\section{Effect of intravitreal ranibizumab in avascular pigment epithelial detachment}

\begin{abstract}
Purpose To evaluate long-term morphologic and functional changes after intravitreal ranibizumab in avascular pigment epithelial detachment (PED) secondary to age-related macular degeneration (AMD).

Patients and methods Interventional, prospective case series; the first group of six patients received three and the second group of six patients received six intravitreal injections of ranibizumab $(0.5 \mathrm{mg})$ at monthly intervals. Outcome measures included the change of PED and retinal volume as determined by spectral domain optical coherence tomography (Cirrus), best-corrected visual acuity (BCVA; Early Treatment Diabetic Retinopathy Study), and macular sensitivity using microperimetry (MP-1; Nidek Co. Ltd). Results The mean baseline PED volume of $1.33 \mathrm{~mm}^{3}$ decreased significantly by $42 \%$ at month $6\left(-0.55 \mathrm{~mm}^{3}, P<0.05\right)$. Compared to baseline no significant change was observed at months 9 and 12. BCVA, retinal volume, and macular sensitivity remained stable during the entire follow-up. In one case a tear of the retinal pigment epithelium was observed after five injections with a consequent decrease of BCVA of four lines.

Conclusion Treatment with intravitreal injections of ranibizumab may temporarily decrease the volume of avascular PED secondary to AMD, however this effect was not maintained over the 1-year study period. The treatment was ineffective for improving retinal function as measured with BCVA and microperimetry. Eye (2010) 24, 962-968; doi:10.1038/eye.2009.265; published online 13 November 2009
\end{abstract}

Received: 22 April 2009 Accepted in revised form: 18 September 2009 Published online: 13 November 2009
Keywords: age-related macular degeneration; optical coherence tomography; pigment epithelial detachment; ranibizumab; VEGF
M Ritter, M Bolz, S Sacu, GG Deák, C Kiss, C Pruente and UM Schmidt-Erfurth

\section{Introduction}

Retinal pigment epithelial detachment (PED) may occur under various conditions in association with age-related macular degeneration (AMD) and contribute to the loss of central vision due to retinal and retinal pigment epithelium (RPE) atrophy and eventually tearing of the RPE. ${ }^{1}$ Studies have indicated that the prognosis in eyes with PED is probably related to the presence of associated choroidal neovascularization $(\mathrm{CNV}){ }^{2}$ It has been shown that the overall risk for developing $\mathrm{CNV}$ within 2 years after diagnosis of serous PED associated with AMD is $24-34 \% .^{2-4}$

Several anti-VEGF treatments have been used to treat primary CNV associated with AMD and have provided significant improvements in maintaining and improving visual function., Ranibizumab (Lucentis; Genetech Inc., South San Francisco, CA, USA) is a recombinant humanized Fab fragment of a monoclonal antibody that has been shown to be an effective inhibitor of all isoforms of VEGF. $^{7,8}$

Optical coherence tomography (OCT) is of particular importance in evaluating effects of anti-VEGF therapies as the modality is able to identify morphologic changes in the epiretinal intra- and subretinal compartments. ${ }^{9}$ Spectral domain OCT (SD-OCT) delineates the configuration of pathologies at the level of the RPE topographically, localizes lesions precisely, and quantifies their extension. By raster scanning together with segmentation of an extrapolated, ideal 'physiologic' RPE demarcation and the realistic pathologic RPE contour including RPE detachments, the volume of the entire PED, and changes under therapy can be quantified. 
There are, as yet, no established treatment recommendations for PED, whether or not associated with CNV. In general, treatment of choroidal neovascular lesions associated with a significant PED was found to be particularly difficult, especially because of the risk of an RPE tear and a presumably limited therapeutic benefit. ${ }^{10-14}$ Eyes with substantial PED components, for example larger than the $\mathrm{CNV}$, were routinely excluded from clinical trials. However, in clinical practice patients often present with PED and the natural course of such lesions is generally poor, so that an effective treatment is clearly needed.

As the main mechanism of action of anti-VEGF therapy is anti-permeability and resolution of intra- and subretinal fluid in exudative AMD, the aim of this prospective study was to investigate the efficacy and safety of intravitreal ranibizumab therapy in serous, non-vascularized PED identified by fluorescein (FA) and indocyanine green angiography (ICGA). Spectral domain, raster scanning OCT was applied as an optimal mode to measure precisely the therapeutic effects by a quantitative analysis of PED volume, and other morphologic parameters, such as retinal volume changes. Simultaneously, functional parameters were determined in detail using a standardized test of central visual function, that is best-corrected visual acuity (BCVA), and a mapping of retinal function over the entire macular area by microperimetry was evaluated.

\section{Materials and methods}

This prospective interventional pilot study was performed at the Department of Ophthalmology at the Medical University of Vienna and enrolled 12 consecutive patients (12 eyes). The study followed the tenets of the Declaration of Helsinki Principles. The study and the study procedures were approved by the local ethics committee at the Medical University of Vienna, registered at the European clinical database (EUDRACT-2007-007655-14). An informed consent was obtained from all patients detailing the potential risks and benefits of the intervention.

The diagnosis of PED secondary to AMD was established by fundoscopy and SD-OCT. In addition, all eyes showed absence of any characteristic signs typical for $\mathrm{CNV}$, retinal angiomatous proliferation, or polypoidal choroid vasculopathy in FA and ICGA. FA was repeated at 3-month intervals to evaluate an eventual development of CNV during the 12-month follow-up. Eyes with features of a 'notch' in serous PED, which was suggested by Sato et $a l^{15}$ as being diagnostically important for the presence of a CNV, were excluded.

According to protocol, BCVA using Early Treatment Diabetic Retinopathy Study (ETDRS) charts had to be
20/400 or higher in the study eye. Patients with isolated PED and no CNV lesion were included in a consecutive manner. The first group of six patients received three and the second group of 6 patients received 6 consecutive intravitreal injections of $0.5 \mathrm{mg}$ ranibizumab at monthly intervals. Injections were administered under sterile conditions in the operating room according to the national recommendations. ${ }^{16}$

A standardized protocol was used to assess morphologic and functional parameters. All tests were carried out by certified study personal.

For evaluation of changes in PED and retinal volumes, SD-OCT, Software 2.0 (Cirrus prototype; Carl Zeiss Meditec, Dublin, CA, USA) was performed at baseline, months 1, 2, 3, 4, 5, 6, 9 and 12. In contrast to the standard time-domain technology used by conventional Stratus OCT, the spectral domain technology of the prototype allowed the acquisition of a complete three-dimensional data set of the posterior pole $(6 \times 6 \mathrm{~mm})$ with an axial resolution of $5 \mu \mathrm{m}$.

At the same intervals, functional retinal changes were documented by assessing BCVA. Microperimetry examination was performed using the Micro Perimeter MP-1 (Nidek Co. Ltd, Padova, Italy) as this technique provides a fully automated and reproducible assessment of central macular function. A static threshold perimetry of the centre and two circles at 3.5 and $7.9^{\circ}$ using a Goldmann size III stimulus was selected and performed in all patients and mean values were calculated.

The paired $t$-test was used for statistic analysis of changes in PED volume, retinal volume, BCVA, and retinal threshold sensitivity compared to baseline. $P \leqslant 0.05$ was considered statistically significant. For correlations, the Pearson's test was used and specified by coefficient ' $r$ ' and by ' $P$ ' for significance.

\section{Results}

Patient characteristics are summarized in Table 1. The follow-up time was 12 months. Of the 12 patients, 2 were withdrawn from the study at month 2 . One of these patients had lost to follow-up due to advanced abdominal aortic aneurysm and the second one refused further treatment.

Most of the eyes presented with a serous PED (7 of 12), whereas 5 were graded as drusenoid PED. Each patient in the first group received three initial intravitreal injections of ranibizumab at monthly intervals, subsequently six individuals received three additional monthly injections thereafter, as a treatment effect by means of a decrease in PED volume had been observed in four of six individuals of the first group.

At baseline, the mean PED volume measured with HD-OCT was $1.33 \mathrm{~mm}^{3}$, ranging from 0.1 to $4.0 \mathrm{~mm}^{3}$. 

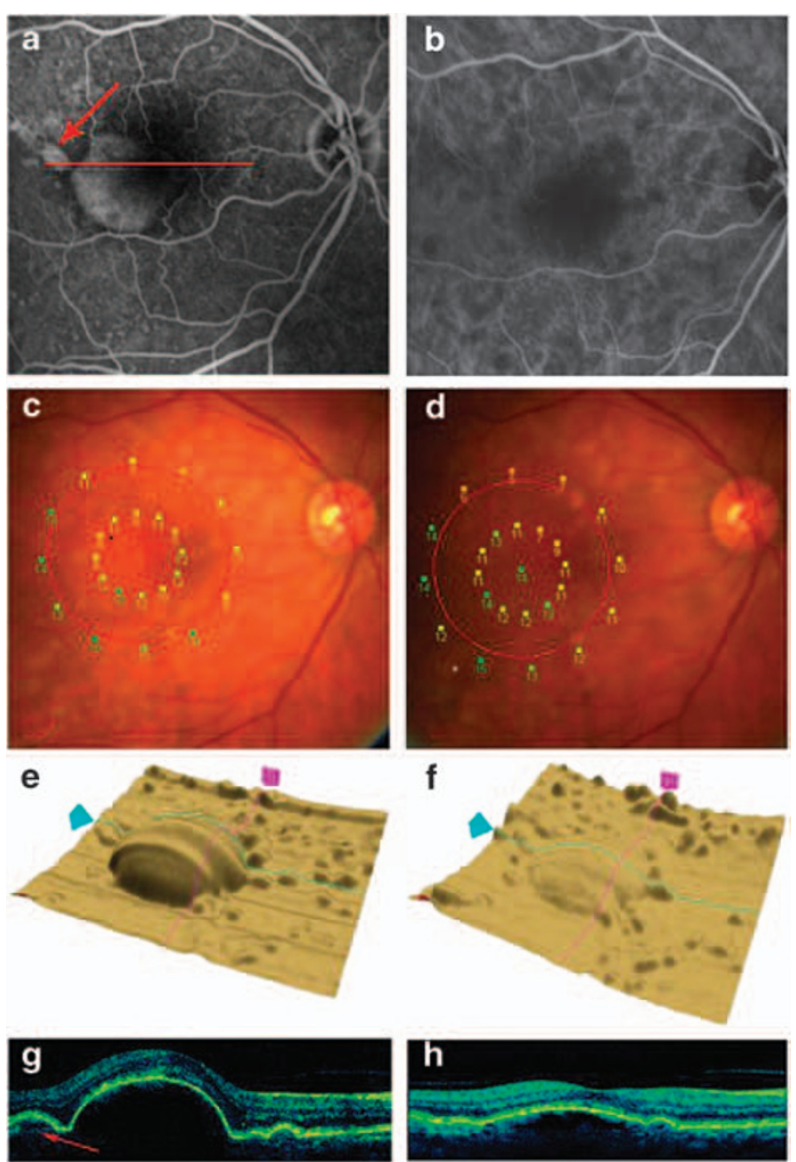

Figure 1 Case 7 (serous PED, treated with six intravitreal injections of ranibizumab). The $5 \mathrm{~min}$ baseline FLA (a) shows fluorescein pooling but no visible signs of CNV. The 5 min ICGA (b) reveals a characteristic hypofluorescence, the satellite lesion seen by FA (arrow) remains silent excluding a notch-like CNV. Microperimetry examination at baseline (c) and month 12 (d) shows a discrete improvement in mean retinal sensitivity by $+1.1 \mathrm{~dB}$. Three-dimensional image of the RPE was generated from $512 \times 128$ axial raster scans at baseline (e; volume $1.7 \mathrm{~mm}^{3}$ ) and month 12 (f; volume $0.3 \mathrm{~mm}^{3}$ ). The A scan at baseline $(\mathrm{g})$ and month 12 (h) highlights the flattening of the PED and the overlying retina with a re-shaping of the foveal contour at month 12. It is particularly noteworthy that the integrity of the outer/inner photoreceptor segment layer appears morphologically restored at month 12 after the flattening of the PED. The detachment is clearly caused by fluid only, at the main lesion site as well as the satellite lesion (arrow), excluding a neovascular component.

At their final visit, four eyes showed a decrease in PED volume by a mean of $56 \%$ (Figure 1) and two PED had resolved completely within the follow-up period (Figure 2). Of these six patients, two had received six injections and four had received three injections, two were graded as drusenoid PED and four as serous PED. In four patients an increase in PED volume by a mean of $33 \%$ was observed at month 12 (Figure 3). Of these eyes, 

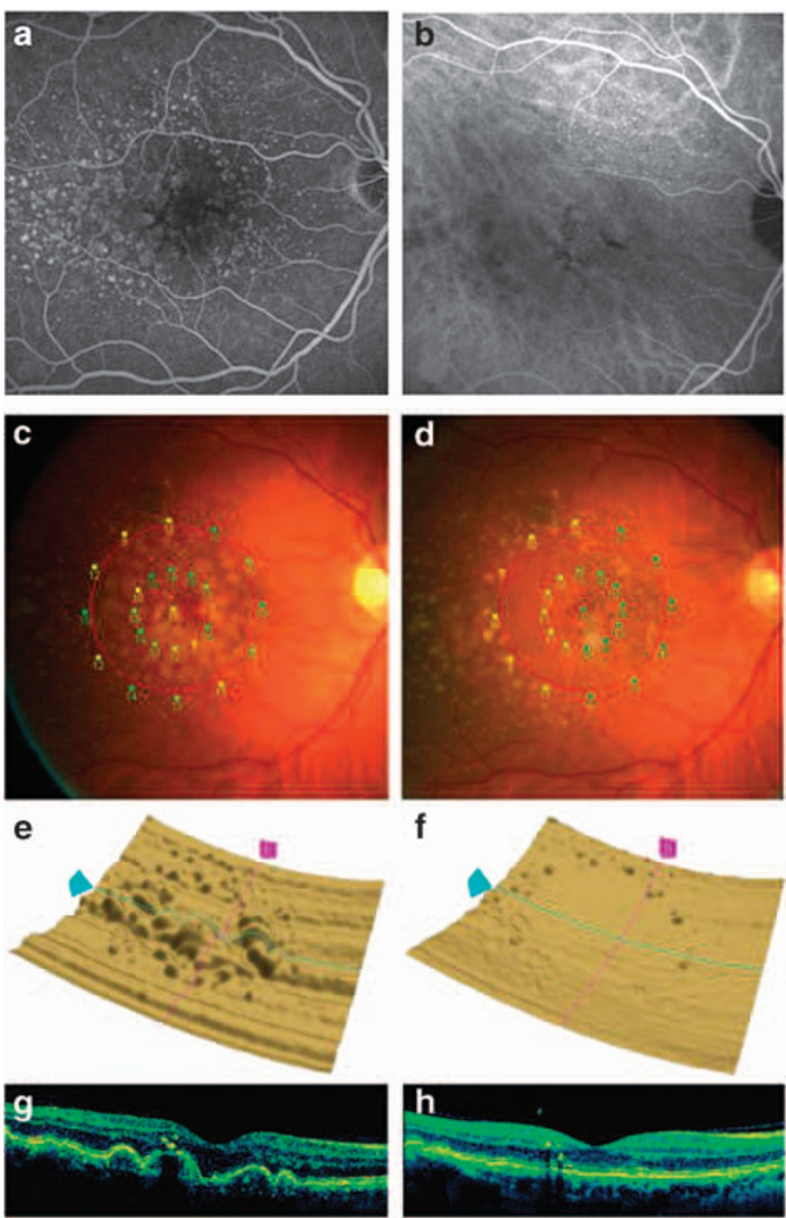

Figure 2 Case 6 (drusenoid PED, treated with three intravitreal injections of ranibizumab). Baseline FLA (a) and ICGA (b) show confluent serous PED from soft drusen with associated RPE hyperpigmentation. Microperimetry examination at baseline (c) and month 12 (d) reveals a stable mean macular sensitivity. The three-dimensional image of the RPE generated from $512 \times 128$ raster axial scans at baseline (e; volume: $0.2 \mathrm{~mm}^{3}$ ) and month 12 (f; volume: $0 \mathrm{~mm}^{3}$ ) shows a complete resolution of sub-RPE fluid deposits with a flattening of the central RPE layer. The scan at baseline $(\mathrm{g})$ and month $12(\mathrm{~h})$ shows disappearance of the focal PED lesions resulting in a regular contour of the intraretinal layers including the fovea and particularly the outer/inner segment and RPE layers.

three received six injections and one received three. In two patients the PED volume remained unchanged, one had received six and the other only three injections.

The variability in clinical and morphologic changes seen under therapy was rather large (Figures 1-3). Although prominent PED flattened progressively with a visible restoration of the layering of the overlying retina and reformation of the foveal depression (Figure 1), a similarly impressive serous PED in another eye failed to show any change in the configuration of the PED (Figure 3). Multiple confluent
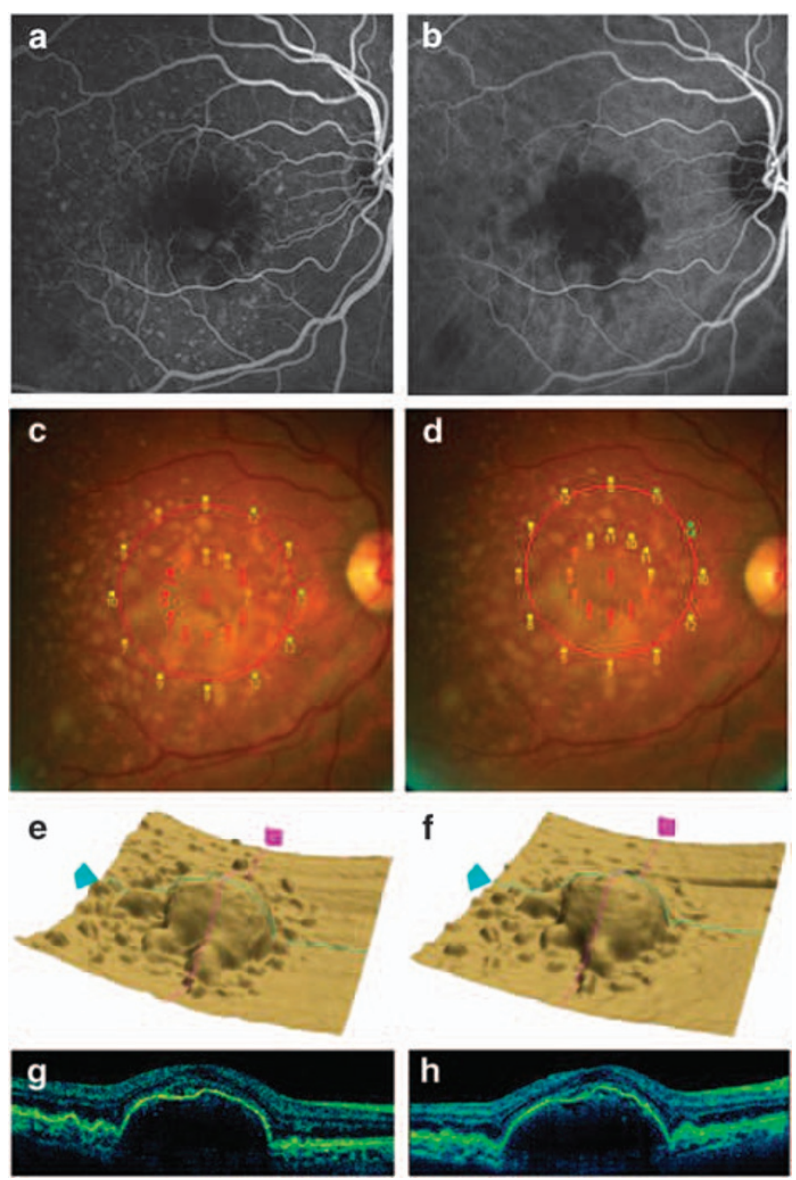

Figure 3 Case 11 (drusenoid PED, treated with six intravitreal injections of ranibizumab): $5 \mathrm{~min}$ baseline FLA (a), 5 min ICG (b). Microperimetry examination at baseline (c) and month 12 (d). Three-dimensional image of the RPE generated from $512 \times 128$ raster axial scans at baseline (e; volume: $1 \mathrm{~mm}^{3}$ ) and month 12 (f; volume: $1.3 \mathrm{~mm}^{3}$ ). Cirrus OCT A scan at baseline $(\mathrm{g})$ and month 12 (h).

smaller PED lesions disappeared completely leaving a smooth RPE surface and a regular retinal layer architecture (Figure 2).

Spectral domain optical coherence tomography measurements showed a mean reduction of PED volume by $10 \%\left(-0.14 \mathrm{~mm}^{3}, P=0.27\right)$ at month 1 , by $21 \%$ $\left(-0.28 \mathrm{~mm}^{3}, P=0.10\right)$ at month 2 , by $31 \%\left(-0.4 \mathrm{~mm}^{3}\right.$, $P=0.10)$ at month 3 , and by $28 \%\left(-0.37 \mathrm{~mm}^{3}, P=0.10\right)$ at month 4 , respectively compared with baseline values. A statistical significant decrease was reached only at month 5 with a reduction by $39 \%\left(-0.51 \mathrm{~mm}^{3}, P<0.04\right)$ and at month 6 with a reduction by $42 \%\left(-0.55 \mathrm{~mm}^{3}\right.$, $P<0.05)$. At month 9, the mean PED volume remained decreased by $41 \%\left(-0.54 \mathrm{~mm}^{3}, P=0.06\right)$, whereas at month 12 the mean decrease was reduced to $25 \%$ $\left(-0.33 \mathrm{~mm}^{3}, P=0.30\right)$ without statistical significance when compared to baseline. 
Mean retinal volume remained stable during the study period with $10.1 \pm 0.59 \mathrm{~mm}^{3}$ at baseline $9.9 \pm 0.65 \mathrm{~mm}^{3}$ at month $3,10.0 \pm 0.64 \mathrm{~mm}^{3}$ at month $6,10.1 \pm 0.63 \mathrm{~mm}^{3}$ at month 9 , and $10.1 \pm 0.9 \mathrm{~mm}^{3}$ at month 12 .

At baseline, mean BCVA was 75.5 ETDRS letters ranging from 58 to 89 letters. Mean BCVA at month 3 (77.1 \pm 9.9$)$, month 6 (75.2 \pm 14.5$)$, month 9 (74.5 \pm 13.8$)$, and month $12(72.5 \pm 15.4)$ did not change significantly from baseline. One eye (case 9) had a severe decrease in BCVA of four lines (22 letters) at month 5 after five injections, due to a tear of the RPE affecting the fovea. The rip occurred after four uneventful injections with a stable PED volume over 4 months and no signs of imminent ruptures. Following the rip the RPE flattened.

The microperimetry assessment of central retinal threshold sensitivity showed stable values over the entire course of the study period. There was no significant fluctuation from $9.8 \pm 3.5 \mathrm{~dB}$ at baseline to $10.1 \pm 3.4 \mathrm{~dB}$ at month 3, $9.7 \pm 3.5 \mathrm{~dB}$ at month $6,9.4 \pm 3.9 \mathrm{~dB}$ at month 9 , and $9.7 \pm 3.2 \mathrm{~dB}$ at month 12 .

There was no statistical significant correlation between the PED volume at baseline and change in PED volume after therapy $(r=0.16 ; P=0.66)$. Furthermore, no significant correlation of baseline PED volume and change in BCVA $(r=-0.27 ; P=0.45)$ or change in macular sensitivity $(r=0.41 ; P=0.24)$ was found.

Throughout the entire follow-up of 12 months, none of the participants showed any ocular adverse event related to the therapy except for the RPE rip, which occurred in a stable PED after several uneventful treatments. None showed signs of a newly developed CNV documented by FA imaging and no systemic complications could be documented.

\section{Conclusion}

The development and natural course of PED in AMD has not yet been fully clarified. It probably is a continuum of degenerative changes occurring secondary to accumulation of 'waste' products and deposition of lipids in Bruch's membrane and formation of CNV. ${ }^{17,18} \mathrm{~A}$ widely accepted hypothesis on the pathogenesis of PED is the reduced hydraulic conductivity of the Bruch's membrane-choroid complex in AMD, which implies a decreased capacity for the exchange of fluid between the choroidal and retinal pigment epithelial compartments. ${ }^{19}$ Several studies indicate that local inflammatory mechanisms contribute to the damage of degenerated RPE and degradation of Bruch's membrane. ${ }^{20}$ Inflammatory mediators and cytokines such as interleukins and complement factors 3 and 5 as well as VEGF secreted by activated phagocytes and other inflammatory cells might be involved in permeability changes in the subretinal and sub-RPE space. ${ }^{21,22}$
Drusenoid and serous PED are two different clinical entities of non-vascularized PED types associated with AMD, but have probably a similar pathogenesis. Drusen are deposits of neutral lipid within the extracellular material of Bruch's membrane, external to the basement membrane of the RPE. ${ }^{23}$ Confluence of drusen, usually occurring in the central macula, probably predisposes to formation of drusenoid PED. ${ }^{24}$ Serous PED is defined as an area of sharply demarcated dome-shaped RPE elevation showing a bright, uniform filling of the entire lesion on FA but without leakage. ${ }^{25}$

In this pilot study, intravitreal injections appeared effective in decreasing the PED volume in 6 of 12 eyes with avascular PED secondary to AMD. However, a significant reduction in mean PED volume could be observed only at months 5 and 6 . This indicates that the treatment response in PED lesions may be slower when compared with regression of CNV alone or smaller PED associated with CNV after anti-VEGF therapy, where a significant reduction of mean PED height could be observed already at 1 week after the first drug administration. ${ }^{26}$ In addition, this limited and prolonged effect seems to be transient and not maintained over a longer period of time after treatment, as the mean PED volume reduction did not reach statistical significance at months 9 and 12. Interestingly, subgroup analysis about a decrease in PED volume showed no difference between patients who received three or six injections, nor between patients with serous or drusenoid PED at baseline. Obviously, anti-VEGF therapy using ranibizumab does not lead to a substantial and predictable treatment effect in this patient population. The limited and variable changes rather suggest a predominance of the spontaneous course of PED disease. In a study by Hartnett et $a l^{4}$ evaluating the natural course, avascular PED associated with drusen showed the best visual prognosis and anatomic results. The natural progression included flattening of the PED in 15 of 21 eyes within 41 months. Neovascularization was found to be rare in the confluent drusen group, whereas $34 \%$ of the serous PED developed CNV over an average follow-up of 25 months. In this study no patient showed signs of a newly developed CNV during the follow-up period. However the follow-up in this study only includes a 12-month period.

Tears of the RPE are reported to occur in $10 \%$ of patients with serous PED secondary to AMD. ${ }^{27}$ They occur spontaneously, ${ }^{1}$ but events after intravitreal injections with ranibizumab have been described as well. ${ }^{28}$ In our report, one RPE tear was observed in a case with a serous PED after six injections with a consecutive decrease of BCVA of four lines. However, it remains unclear, whether this RPE rip was caused by the treatment per se or reflects the $10 \%$ rate of spontaneous events. 
Retinal volume was measured to identify an effect on the neurosensory layers after changes in the underlying RPE compartment, such as an increase or decrease of intra- and subretinal fluid or retinal atrophy. It remained stable without statistically significant changes during the 1-year follow-up. No functional improvement in terms of an increase in BCVA or macular sensitivity could be observed over the course of the study duration. These results may be influenced by the relatively high level of BCVA and macular sensitivity at baseline, which is a common finding in these pathologies. Nevertheless, it may be suggested that long-standing PED and thus impaired nutrition to the RPE and photoreceptor cells has already resulted in irreversible damage. This is supported by the finding that no correlation could be found between a reduction in PED volume and functional parameters, although, in some eyes, a scan imaging appeared to show a restoration of the integrity of outer retinal layers.

Generally, SD-OCT proved to be a valuable tool to image morphologic features of retinal disease in nonvascular PED. Owing to the all location raster mode, it is the first imaging technique allowing for a precise quantification of PED volume and retinal volume, which can be used for a reliable follow-up during anti-VEGF therapy.

In addition, this is the first report of the effect of intravitreal injections with ranibizumab for treatment of avascular PED secondary to AMD using a prospective standardized protocol. In conclusion, these data suggest a primary involvement of VEGF in the pathophysiology of PED even in cases without angiographic signs of a CNV. Therapy with ranibizumab may have an effect on temporarily decreasing the volume of PED secondary to AMD, in a slowly progressive pattern but no sustained effect over the 1-year observation period without re-injections could be observed. Even if the specific role of VEGF in serous/drusenoid non-vascularized PED is not known, we suspect this reduction of PED volume to be mediated by the anti-permeability and antiinflammatory effects of ranibizumab. Importantly, this morphologic effect seems to be irrelevant for improving retinal function in the foveal center or the overall macular area as measured with BCVA and microperimetry.

It has to be considered that these results are based on an interventional, uncontrolled case series including a limited number of patients. As the effect of ranibizumab on non-vascular PED may be intrinsic smaller compared to PED in other forms of neovascular AMD, larger studies evaluating this question are needed. Such investigation is also needed to identify possible side effects such as RPE rips, which are a pathognomonic finding during the natural course of the disease.
Summary

What was known before

- Ranibizumab has been used to treat primary CNV associated with AMD and has provided significant improvements in maintaining and improving visual function.

What this study adds

- Therapy with ranibizumab may have an effect on temporarily decreasing the volume of non-vascularized PED secondary to AMD.

\section{Conflict of interest}

The authors declare no conflict of interest.

\section{References}

1 Decker WL, Sanborn GE, Ridley M, Annesley Jr WH, Sorr EM. Retinal pigment epithelial tears. Ophthalmology 1983; 90: 507-512.

2 Klein ML, Obertynski H, Patz A, Fine SL, Kini M. Follow-up study of detachment of the retinal pigment epithelium. $\mathrm{Br} J$ Ophthalmol 1980; 64: 412-416.

3 Elman MJ, Fine SL, Murphy RP, Patz A, Auer C. The natural history of serous retinal pigment epithelium detachment in patients with age-related macular degeneration. Ophthalmology 1986; 93: 224-230.

4 Hartnett ME, Weiter JJ, Garsd A, Jalkh AE. Classification of retinal pigment epithelial detachments associated with drusen. Graefes Arch Clin Exp Ophthalmol 1992; 230: 11-19.

5 Heier JS, Antoszyk AN, Pavan PR, Leff SR, Rosenfeld PJ, Ciulla TA et al. Ranibizumab for treatment of neovascular age-related macular degeneration: a phase I/II multicenter, controlled, multidose study. Ophthalmology 2006; 113 : 642e1-642e4.

6 Spaide RF, Laud K, Fine HF, Klancnik Jr JM, Meyerle CB, Yannuzzi LA et al. Intravitreal bevacizumab treatment of choroidal neovascularization secondary to age-related macular degeneration. Retina 2006; 26: 383-390.

7 Krzystolik MG, Afshari MA, Adamis AP, Gaudreault J, Gragoudas ES, Michaud NA et al. Prevention of experimental choroidal neovascularisation with intravitreal anti-vascular endothelial growth factor antibody fragment. Arch Ophthalmol 2002; 120: 338-346.

8 Rosenfeld PJ, Rich RM, Lalwani GA. Ranibizumab: phase III clinical trial results. Ophthalmol Clin North Am 2006; 19: 361-372.

9 Rosenfeld PJ, Moshfeghi AA, Puliafito CA. Optical coherence tomography findings after an intravitreal injection of bevacizumab (Avastin) for neovascular agerelated macular degeneration. Ophthalmic Surg Lasers Imaging 2005; 36: 331-335.

10 Lim JI, Aaberg TM, Capone Jr A, Sternberg Jr P. Indocyanine green angiography-guided photocoagulation of choroidal neovascularization associated with retinal pigment epithelial detachment. Am J Ophthalmol 1997; 123: 524-532.

11 Gross-Jendroska M, Flaxel CJ, Schwartz SD, Holz FG, Fitzke FW, Gabel VP et al. Treatment of pigment epithelial detachments due to age-related macular degeneration with 
intra-ocular C3F8 injection. ANZ J Ophthalmol 1998; 26: 311-317.

12 Axer-Siegel R, Ehrlich R, Rosenblatt I, Kramer M, Priel E, Yassur Y et al. Photodynamic therapy for occult choroidal neovascularization with pigment epithelium detachment in age-related macular degeneration. Arch Ophthalmol 2004; 122: $453-459$.

13 Nicoló M, Ghiglione D, Lai S, Calabria G. Intravitreal triamcinolone in the treatment of serous pigment epithelial detachment and occult choroidal neovascularization secondary to age-related macular degeneration. Eur J Ophthalmol 2005; 15: 415-419.

14 Dhalla MS, Blinder KJ, Tewari A, Hariprasad SM, Apte RS Retinal pigment epithelial tear following intravitreal pegaptanib sodium. Am J Ophthalmol 2006; 141: 752-754.

15 Sato T, Iida T, Hagimura N, Kishi S. Correlation of optical coherence tomography with angiography in retinal pigment epithelial detachment associated with age-related macular degeneration. Retina 2004; 24: 910-914.

16 Jaissle GB, Szurman P, Bartz-Schmidt KU. Recommendation for the implementation of intravitreal injections - statement of the German Retina Society, the German Society of Ophthalmology (DOG) and the German Professional Association of Ophthalmologists (BVA). Klin Monatsbl Augenheilkd 2005; 222: 390-395.

17 Bird AC, Marshall J. Retinal pigment epithelial detachments in the elderly. Trans Ophthalmol Soc UK 1986; 105: 674-682.

18 Holz FG, Pauleikhoff D, Klein R, Bird AC. Pathogenesis of lesions in late age-related macular disease. Am J Ophthalmol 2004; 137: 504-510.

19 Moore DJ, Hussain AA, Marshall J. Age-related variation in the hydraulic conductivity of Bruch's membrane. Invest Ophthalmol Vis Sci 1995; 36: 1290-1297.
20 Hollyfield JG, Bonilha VL, Rayborn ME, Yang X, Shadrach $\mathrm{KG}$, Lu L et al. Oxidative damage-induced inflammation initiates age-related macular degeneration. Nat Med 2008; 14: 194-198.

21 Hageman GS, Luthert PJ, Victor Chong NH, Johnson LV, Anderson DH, Mullins RF. An integrated hypothesis that considers drusen as biomarkers of immune-mediated processes at the RPE-Bruch's membrane interface in aging and age-related macular degeneration. Prog Retin Eye Res 2001; 20: 705-732.

22 Oh H, Takagi H, Takagi C, Suzuma K, Otani A, Ishida K et al. The potential angiogenic role of macrophages in the formation of choroidal neovascular membranes. Invest Ophthalmol Vis Sci 1999; 40: 1891-1898.

23 Pauleikhoff D, Harper CA, Marshall J, Bird AC. Aging changes in Bruch's membrane. A histochemical and morphologic study. Ophthalmology 1990; 97: 171-178.

24 Casswell AG, Kohen D, Bird AC. Retinal pigment epithelial detachments in the elderly: classification and outcome. $\mathrm{Br} \mathrm{J}$ Ophthalmol 1985; 69: 397-403.

25 Gass JDM. Stereoscopic Atlas of Macular Disease: Diagnosis and Management. 4th ed. Mosby: St Louis, 1997, pp 24-26 and 82-87.

26 Bolz M, Michels S, Geitzenauer W, Prager F, SchmidtErfurth U. Effect of systemic bevacizumab therapy on retinal pigment epithelial detachment. Br J Ophthalmol 2007; 91: 785-789.

27 Casswell AG, Kohen D, Bird AC. Retinal pigment epithelial detachments in the elderly: classification and outcome. Br J Ophthalmol 1985; 69: 397-403.

28 Bakri SJ, Kitzmann AS. Retinal pigment epithelial tear after intravitreal ranibizumab. Am J Ophthalmol 2007; 143: 505-507. 